\title{
Editorial: Genetics of Age-Related Diseases and Their Risk and Protective Factors
}

\author{
Ida K. Karlsson ${ }^{1,2}$, Thalida Em Arpawong ${ }^{3}$, Yiqiang Zhan $^{4 *}$ and Kelli Lehto ${ }^{5}$ \\ ${ }^{1}$ Department of Medical Epidemiology and Biostatistics, Karolinska Institutet, Stockholm, Sweden, ${ }^{2}$ Aging Research Network- \\ Jönköping, School of Health and Welfare, Jönköping University, Jönköping, Sweden, ${ }^{3}$ Leonard Davis School of Gerontology, \\ University of Southern California, Los Angeles, CA, United States, ${ }^{4}$ School of Public Health, Sun Yat-Sen University, Guangzhou, \\ China, ${ }^{5}$ Institute of Genomics, University of Tartu, Tartu, Estonia
}

Keywords: genetic epidemiology, aging, age-related disease, editorial, risk factor, protective factor

Editorial on the Research Topic

Genetics of Age-Related Diseases and Their Risk and Protective Factors

Aging is a universal biological process, but with great individual variability, where some remain healthy throughout old age while others suffer from health problems and co-morbidities from a relatively early old age (Melzer et al., 2020). Twin studies have estimated the heritability of the common age-related diseases cardiovascular disease, type 2 diabetes, and Alzheimer's disease to between 45 and 80\% (Zdravkovic et al., 2002; Gatz et al., 2006; Willemsen et al., 2015). This indicates that a substantial amount of the total variance in these conditions is attributable to genetic variation, and that genetic study designs can make important contributions to our understanding of aging processes and age-related diseases. Indeed, genome-wide association studies (GWASs) have identified many genetic variants associated with these conditions (Nelson et al., 2017; Mahajan et al., 2018; Jansen et al., 2019), and other age-related diseases, even if they still explain only part of the estimated heritability. Important insights into disease mechanisms have also been made by using the results from GWASs to understand e.g. biological pathways and disease associations (Melzer et al., 2020). The aim of this research topic was to join high quality research projects in genetics that help us to improve our understanding of age-related diseases as well as the role of their risk and protective factors. In this research topic, six Original Research Articles and one Brief Research Report present important and relevant findings within the fields of genetics and age-related diseases.

Urzi and colleagues investigated genetic influences in sarcopenia, a geriatric disease characterized by loss of muscle mass and strength, and leads to impairment of one's physical capacity. The genetic influences of sarcopenia are still poorly understood, and the authors here conducted a pilot study testing the single and combined effects of seven selected single nucleotide polymorphisms (SNPs). They identified statistically significant associations between three of the seven SNPs and sarcopenia, namely MTHFR, ACTN3, and NRF2, and show that these three SNPs together explain a large proportion of the variability in the disease.

It is important not only to understand risk and protective factors of a disease, but also what influences the outcomes of the disease. An excellent example is the study by Dungan et al., who conducted a GWAS in a survival analysis setting, examining genetic variants associated with survival among individuals diagnosed with coronary artery disease (CAD). The authors could highlight two SNPs, one of which is novel and one of which reside in the DAB2IP gene, also implicated in CAD diagnosis (Harst and Verweij, 2018).

Four studies within the topic focused on dementia and Alzheimer's disease (AD). Kim et al. used a twin sample to examine the measured (APOE genotype) and unmeasured (twin design) 
genetic effects underlying the relationship between age-related increase in loneliness and dementia risk. The authors showed that although the baseline level, but not change in loneliness, predicted dementia, these associations diminished after adjusting for genetic and environmental confounds.

Three of the articles used a Mendelian randomization (MR) approach to understand causal influences on the risk of dementia. Ware and colleagues used individual-level data from the U.S. Health and Retirement Study in a one-sample MR design, examining causal effects of type- 2 diabetes on the risk of cognitive impairment and dementia. While type- 2 diabetes was associated with cognitive impairment, but not dementia, the MR analyses indicated that the association is not of a causal nature. This is in agreement with previous two-sample MR studies of type-2 diabetes and AD, based on GWAS summary statistics (Østergaard et al., 2015; Walter et al., 2016; Thomassen et al., 2020), and the study makes an important contribution by demonstrating that the lack of causality is seen also for cognitive impairment, and is robust to adjustment for education and $A P O E \& 4$ status.

$\mathrm{Yu}$ et al. used summary-level data from GWAS to conduct a two-sample MR study demonstrating that shorter telomere length, a marker of biological aging, is causally associated with risk of $\mathrm{AD}$. This strengthens previous findings on the topic (Zhan et al., 2015; Gao et al., 2019; Guo and Yu, 2019), extending the analyses to a larger sample size and using additional methods to test the robustness of the causal association.

In a Brief Research Report, $\mathrm{Wu}$ and colleagues examined the association between growth differentiation factor 15 (GDF15) and neurodegenerative diseases in a two-sample MR study. The authors found GDF-15 to be causally associated with $\mathrm{AD}$, but not with Parkinson's disease or amyotrophic

\section{REFERENCES}

Gao, K., Wei, C., Zhu, J., Wang, X., Chen, G., Luo, Y., et al. (2019). Exploring the Causal Pathway from Telomere Length to Alzheimer's Disease: An Update Mendelian Randomization Study. Front. Psychiatry 10, 843. doi:10.3389/ fpsyt.2019.00843

Gatz, M., Reynolds, C. A., Fratiglioni, L., Johansson, B., Mortimer, J. A., Berg, S., et al. (2006). Role of Genes and Environments for Explaining Alzheimer Disease. Arch. Gen. Psychiatry 63 (2), 168-174. doi:10.1001/archpsyc.63.2.168

Guo, Y., and Yu, H. (2019). Leukocyte Telomere Length Shortening and Alzheimer's Disease Etiology. J. Alzheimers Dis. 69 (3), 881-885. doi:10.3233/jad-190134

Harst, P. V. D., and Verweij, N. (2018). Identification of 64 Novel Genetic Loci Provides an Expanded View on the Genetic Architecture of Coronary Artery Disease. Circ. Res. 122 (3), 433-443. doi:10.1161/ CIRCRESAHA.117.312086

Jansen, I. E., Savage, J. E., Watanabe, K., Bryois, J., Williams, D. M., Steinberg, S., et al. (2019). Genome-wide Meta-Analysis Identifies New Loci and Functional Pathways Influencing Alzheimer's Disease Risk. Nat. Genet. 51, 404. doi:10.1038/s41588-018-0311-9

Mahajan, A., Taliun, D., Thurner, M., Robertson, N. R., Torres, J. M., Rayner, N. W., et al. (2018). Fine-mapping Type 2 Diabetes Loci to Single-Variant Resolution Using High-Density Imputation and Islet-specific Epigenome Maps. Nat. Genet. 50 (11), 1505-1513. doi:10.1038/s41588-018-0241-6 lateral sclerosis. GDF-15 is involved in inflammation and proposed to be associated with healthy aging and age-related diseases, and the findings presented by $\mathrm{Wu}$ et al. indicate that it may be a promising diagnostic marker and therapeutic target for $\mathrm{AD}$.

Genetic research methods are constantly improving, a good example of which is presented in the Research Article by Gao and colleagues. Here, the authors demonstrate that sex-specific twosample MR studies may be biased if sex heterogeneity in the instrumental variable is not considered, i.e. when the instrumental variable is based on results from men and women combined, while the outcome is sex-specific and based on results from only men or only women. This is demonstrated by examining the causal effects of anthropometric traits on breast and prostate cancer, indicating that instrumental variables based on sex-combined summary data may be biased relative to those based on sex-stratified data.

In summary, the Research Topic "Genetics of Age-Related Diseases and Their Risk and Protective Factors" has brought together articles covering broad and important areas within genetics of age-related diseases. Importantly, the articles demonstrate how genetic methods can be used to advance our understanding of disease mechanisms and provide targets for future hypothesis-driven studies, as well as how to make use of already existing knowledge, data, and findings to better understand how risk and protective factors act to influence etiologies for age-related diseases.

\section{AUTHOR CONTRIBUTIONS}

All authors listed have made a substantial, direct, and intellectual contribution to the work and approved it for publication.

Melzer, D., Pilling, L. C., and Ferrucci, L. (2020). The Genetics of Human Ageing. Nat. Rev. Genet. 21 (2), 88-101. doi:10.1038/s41576-019-0183-6

Nelson, C. P., Goel, A., Goel, A., Butterworth, A. S., Kanoni, S., Webb, T. R., et al. (2017). Association Analyses Based on False Discovery Rate Implicate New Loci for Coronary Artery Disease. Nat. Genet. 49 (9), 1385-1391. doi:10.1038/ng.3913

Østergaard, S. D., Mukherjee, S., Sharp, S. J., Proitsi, P., Lotta, L. A., Day, F., et al. (2015). Associations between Potentially Modifiable Risk Factors and Alzheimer Disease: A Mendelian Randomization Study. Plos Med. 12 (6), e1001841. doi:10.1371/journal.pmed.1001841

Thomassen, J. Q., Tolstrup, J. S., Benn, M., and Frikke-Schmidt, R. (2020). Type-2 Diabetes and Risk of Dementia: Observational and Mendelian Randomisation Studies in 1 Million Individuals. Epidemiol. Psychiatr. Sci. 29, e118-e. doi:10.1017/s2045796020000347

Walter, S., Marden, J. R., Kubzansky, L. D., Mayeda, E. R., Crane, P. K., Chang, S.-C., et al. (2016). Diabetic Phenotypes and Late-Life Dementia Risk. Alzheimer Dis. Assoc. Disord. 30 (1), 15-20. doi:10.1097/wad.0000000000000128

Willemsen, G., Ward, K. J., Bell, C. G., Christensen, K., Bowden, J., Dalgård, C., et al. (2015). The Concordance and Heritability of Type 2 Diabetes in 34,166 Twin Pairs from International Twin Registers: The Discordant Twin (DISCOTWIN) Consortium. Twin Res. Hum. Genet. 18 (6), 762-771. doi:10.1017/thg.2015.83

Zdravkovic, S., Wienke, A., Pedersen, N. L., Marenberg, M. E., Yashin, A. I., and De Faire, U. (2002). Heritability of Death from Coronary Heart Disease: a 36-year Follow-Up of 20966 Swedish Twins. J. Intern. Med. 252 (3), 247-254. doi:10.1046/j.1365-2796.2002.01029.x 
Zhan, Y., Song, C., Karlsson, R., Tillander, A., Reynolds, C. A., Pedersen, N. L., et al. (2015). Telomere Length Shortening and Alzheimer Disease-A Mendelian Randomization Study. JAMA Neurol. 72 (10), 1202-1203. doi:10.1001/ jamaneurol.2015.1513

Conflict of Interest: The authors declare that the research was conducted in the absence of any commercial or financial relationships that could be construed as a potential conflict of interest.

Publisher's Note: All claims expressed in this article are solely those of the authors and do not necessarily represent those of their affiliated organizations, or those of the publisher, the editors and the reviewers. Any product that may be evaluated in this article, or claim that may be made by its manufacturer, is not guaranteed or endorsed by the publisher.

Copyright $\odot 2021$ Karlsson, Arpawong, Zhan and Lehto. This is an open-access article distributed under the terms of the Creative Commons Attribution License (CC $B Y)$. The use, distribution or reproduction in other forums is permitted, provided the original author(s) and the copyright owner(s) are credited and that the original publication in this journal is cited, in accordance with accepted academic practice. No use, distribution or reproduction is permitted which does not comply with these terms. 